\title{
Degeneracy in Loop Variables
}

\author{
J. N. Goldberg, J. Lewandowski ${ }^{1}$, and C. Stornaiolo ${ }^{2}$ \\ Department of Physics, Syracuse University, Syracuse, NY 13244-1130, USA \\ 1 Visiting Fulbright Fellow from Instytut Fizyki Teoretycznej, Uniwersytet Warszawski, \\ Hoza 69, PL-00-681 Warszawa, Poland \\ 2 Visiting Scholar from INFN-Sezione Di Napoli, Dipartimento di Scienze Fisiche, \\ I-180125 Napoli, Italy
}

Received October 30, 1991

\begin{abstract}
The small algebra of loop functionals, defined by Rovelli and Smolin, on the Ashtekar phase space of general relativity is studied. Regarded as coordinates on the phase space, the loop functionals become degenerate at certain points. All the degenerate points are found and the corresponding degeneracy is discussed. The intersection of the set of degenerate points with the real slice of the constraint surface is shown to correspond precisely the Goldberg-Kerr solutions. The evolution of the holonomy group of Ashtekar's connection is examined, and the complexification of the holonomy group is shown to be preserved under it. Thus, an observable of the gravitational field is constructed.
\end{abstract}

\section{Introduction}

With the introduction by Ashtekar [1-4] of an $S L(2, \mathbb{C})$ connection $A_{a}{ }^{A}{ }_{B}$ and a densitized triad $\sigma^{a A}{ }_{B}$ as new variables in canonical general relativity, the phase space takes on the appearance of a Yang-Mills gauge theory. A connection as a configuration space variable suggests the using of the parallel transport as the main device to construct gauge invariant quantities. The parallel transport of an arbitrary vector in the spinor space around all closed loops in the base manifold defines the holonomy group of the Yang-Mills connection. Elements of the holonomy group are given by the path ordered exponential of the integral of the connection $A_{a}$ around closed loops. The traces of the holonomy integrals are gauge invariant functionals on the phase space, the Wilson loops. These functionals, identified as $T^{0}$, and the traces of the product of the holonomy integrals and the $\sigma^{a}, T^{1}$ functionals, form a closed Poisson bracket algebra. Therefore, they may be considered to be new configuration and momentum variables, thus new coordinates on the phase space. In fact, the use of these variables as new phase space coordinates was first introduced by Jacobson and Smolin [5] for the Ashtekar phase space for general relativity. Rovelli and Smolin [6] then showed that functionals $T^{n}$ defined by the traces of the product of the holonomy integrals on polynomials homogeneous of degree $n$ in the $\sigma^{a}$ form a graded algebra. 
These functionals have been used in the quantization of $2+1$ gravity [7] and the Maxwell field as test examples [8]. In general relativity, the $T^{n}$-functionals are being studied intensively and it is hoped that the connection and the loop representations of $T^{n}$ 's will lead to a nonperturbative formulation of quantum gravity $[3,5,6,9,10]$.

However, in considering the transition to a quantum theory, there are certain precautions one must take in considering which phase space variables to take over as quantum operators [3]. One wants to choose a set of elementary classical variables whose Poisson bracket algebra is closed and at the same time is large enough to define any sufficiently regular function on the phase space. This is certainly satisfied if the elementary variables are themselves canonically conjugate variables. If they are not, as is the case with the $T^{0}$ and $T^{1}$ variables, there may be functionals of interest which cannot be constructed with the chosen set of elementary variables. In this case the set is incomplete. The set may also be overcomplete in that there may be some algebraic relations among the variables. When this happens, additional care must be taken in the construction of a quantum algebra.

Here we are concerned with the question of completeness of the $T^{0}$ and $T^{1}$ variables. We examine the question raised by Rovelli and Smolin [6] whether given the $T^{0}$ and $T^{1}$ functionals, it is possible, up to a gauge transformation, to recover the connection variables and their canonically conjugate momenta. Phrased another way, are there sets of gauge equivalent points on the phase space of connections and momenta which cannot be recovered uniquely from knowledge of the $T^{0}$ and $T^{1}$ functionals alone? While this question has relevance for all gauge fields, we focus our attention on the group $S L(2, \mathbb{C})$ and the Ashtekar phase space of general relativity. The method of analysis we use can be applied to any other group of interest; e.g., $S U(3)$ used in QCD.

Thus, the aim of our work is to study what information about an $S L(2, \mathbb{C})$ connection $A$ and an $S L(2, \mathbb{C})$-densitized vector field $\sigma$ - both defined on a spatial three manifold $\Sigma$ - is contained in $T^{0}$ and $T^{1}$ functionals and to identify what information is missing. Those pairs $(A, \sigma)$ which cannot be completely recovered (up to a gauge transformation) from $\left(T^{0}, T^{1}\right)$ will be called "degenerate points." We shall find that these degenerate points define surfaces of "measure zero" in the group space.

In Sect. 2 we introduce our notation and recall the precise definitions of $\left(T^{0}, T^{1}\right)$.

Since an important tool in our considerations is the holonomy of the connection, and it is known that any Lie subgroup of the gauge group can be the holonomy group of a particular connection, in Sect. 3 we list real and complex Lie subalgebras of $\operatorname{sl}(2, \mathbb{C})$ (see also [12]).

In Sects. 4 and 5 we study the $T$-functionals in a phase space of complexified gravity, i.e. in the set $\Gamma$ of the $S L(2, \mathbb{C})$-gauge inequivalent pairs $(A, \sigma)$. We find and classify all sets in $\Gamma$, points of which are indistinguishable by $\left(T^{0}, T^{1}\right)$. The general result is that any of those sets contains more than one element if and only if the holonomy group of $A$ is included in the four dimensional subgroup of null rotations (via $\operatorname{sl}(2, \mathbb{C}) \equiv o(3,1, \mathbb{R})$ ). Then there are four kinds of degeneracy depending on the holonomy group and the triad $\sigma$.

In Sect. 6 we formulate a pure $T$-description of degenerate points. We show that sets of points of the same class of degeneracy can be described as solutions to certain equations imposed on $\left(T^{0}, T^{1}\right)$ functionals. We also note that an important feature of degenerate $(A, \sigma)$ is that the connection $A$ admits a covariantly constant spinor direction.

In Sects. 7 and 8 we complete a bridge between $3+1$-Hamiltonian approach in terms of Ashtekar variables and a covariant 4-dimensional formulation of gravity the- 
ory. The task is to relate the holonomy group of the connection $A$ with the holonomy group of the self dual connection of the corresponding space-time endowed with a complex gravitational field. In order to see that relation, we study the Hamiltonian evolution of the holonomy group from initial data. The final result of Sect. 8 consists in finding the intersection between: (i) the set of degenerate points in $\Gamma$ and (ii) the real slice of the constraint surface which corresponds to real vacuum solutions to Einstein's equations. It turns out that the intersection consists of Goldberg-Kerr solutions $[11,12]$.

Another issue, however, is to what extent is degeneracy at $(A, \sigma)$, for $(A, \sigma)$ belonging to the real slice of the constraint surface, also included in this surface. We study this problem in two steps. First, in Sect. 9 we restrict $\left(T^{0}, T^{1}\right)$ to the subspace $\Gamma_{\mathbb{R}}$ of $\Gamma$ which consists of all the configurations $(A, \sigma)$ such that the corresponding 3 -metric is real and its reality is preserved by Hamiltonian evolution. At this level of the argument $(A, \sigma)$ need not satisfy the constraints. It turns out that the reality conditions remove some, but not all of the degeneracy. We construct, in particular, all the configurations $(A, \sigma)$ of the 4-dimensional holonomy group and linearly independent triads such that there still exist in $\Gamma_{\mathbb{R}}$ nontrivial surfaces of those $\left(A^{\prime}, \sigma^{\prime}\right)$ which are indistinguishable from $(A, \sigma)$ using $\left(T^{0}, T^{1}\right)$.

Finally, in Sect.10, we impose the constraints as well as the reality conditions on $(A, \sigma)$ 's. We find the intersection of the surfaces of the same value of $\left(T^{0}, T^{1}\right)$ with the real slice of the constraint surface. We find that the remaining degeneracy is transversal to orbits of the group of transformations generated by the constraints.

To simplify our considerations we treat the 3-manifold $\Sigma$ as $\mathbb{R}^{3}$ or as the 3-sphere $S^{3}$. It implies that the $S L(2, \mathbb{C})$ bundle related to Ashtekar gauge configuration and the holonomy subbundle of $A$ are trivial. It is not difficult, however, to generalize our study to $(A, \sigma)$ which are associated to a $S L(2, \mathbb{C})$-principal fiber bundle over an arbitrary 3-manifold. In particular Theorem 7.1 about the propagation of the holonomy group is true in a general case.

Beginning with Sect. 7 , we require that the triad is linearly independent, i.e. the determinant of the 3-metric tensor is not zero.

\section{Formalism}

Ashtekar variables [1] $A$ and $\sigma$ are defined on a 3-dimensional manifold $\Sigma . A$ is an $\operatorname{sl}(2, \mathbb{C})$ valued differential 1 -form and $\sigma$ is an $\operatorname{sl}(2, \mathbb{C})$ valued vector density of weight 1. Pairs $\left(A^{\prime}, \sigma^{\prime}\right)$ and $(A, \sigma)$ are gauge equivalent if there exists a gauge transformation $a: \Sigma \rightarrow S L(2, \mathbb{C})$ such that

$$
A^{\prime}=a^{-1} A a+a^{-1} d a, \quad \sigma^{\prime}=a^{-1} \sigma a .
$$

The set $\Gamma$ of gauge equivalent classes $[(A, \sigma)]$ can be regarded as a phase space for complexified gravity. It is convenient to introduce a 2-complex-dimensional vector space (a spinor space) endowed with a symplectic form $\varepsilon$, i.e. a bilinear mapping

$$
\varepsilon: V \times V \rightarrow \mathbb{C}
$$

The gauge group of $A$ and $\sigma$ will be considered as the group of endomorphisms of $V$ preserving $\varepsilon$. The 1 -form $A$ defines a parallel transport of a spinor $\lambda \in V$ from a point $x \in \Sigma$ to $y \in \Sigma$ along a given curve $\gamma$. If $\gamma:\left[t_{1}, t_{2}\right] \rightarrow \Sigma$ is a parametrization of $\gamma$ and $\gamma\left(t_{1}\right)=x_{1}, \gamma\left(t_{2}\right)=y$ then

$$
\lambda(y):=h\left(t_{2}, t_{1}\right) \lambda(x),
$$


where an $S L(2, \mathbb{C})$ element $h\left(t_{2}, t_{1}\right)$ is given by the following equation along $\gamma$,

$$
\frac{d}{d t} h\left(t, t_{1}\right)=-A_{a}(\gamma(t)) \dot{\gamma}^{a}(t) h\left(t, t_{1}\right)
$$

with the initial condition

$$
h\left(t_{1}, t_{1}\right)=1 .
$$

If $\gamma\left(t_{2}\right)=\gamma\left(t_{1}\right)=x$, i.e. $\gamma$ is a closed loop, then we denote

$$
h_{\gamma}:=h\left(t_{2}, t_{1}\right) \text {. }
$$

All $h_{\gamma}$ 's associated with a fixed point $x$ constitute a holonomy group $H \subset S L(2, \mathbb{C})$. Given holonomy at $x$, i.e. the mapping $\gamma \rightarrow h_{\gamma}$ for all the closed loops starting and ending at $x$, one can reconstruct the connection $A$ up to gauge transformations. On the other hand, if the holonomy $h_{\gamma}$ at $x$ corresponds to $A$ then holonomy of $A^{\prime}=a^{-1} A a+a^{-1} d a$ is given by $h_{\gamma}^{\prime}=a(x)^{-1} h_{\gamma} a(x)$. Hence $A$ and $A^{\prime}$ possess the same holonomy at $x$ if $a(x)$ commutes with all the elements of the holonomy group at $x$.

Following Rovelli and Smolin [6] we will study mappings $T^{0}$ and $T^{1}$ which label each loop $\gamma$ starting and ending at $x$. The labels $T^{0}$ are $\mathbb{C}$ numbers $T_{\gamma}$,

$$
T_{\gamma}:=\operatorname{Tr} h_{\gamma}
$$

and those of $T^{1}$ are $\mathbb{C}$ valued vector densities of weight $1 T_{\gamma}^{a}$ defined as follows:

$$
T_{\gamma}^{a}(x):=\operatorname{Tr} h_{\gamma} \sigma^{a}(x),
$$

where indices $a$ labels coordinates of tangent vectors and 1-forms with respect to coordinates $\left(x^{a}\right)$ on $\Sigma . T_{\gamma}$ and $T_{\gamma}^{a}$ don't depend on a parametrization of $\gamma$ and they are invariant with respect to gauge transformations.

\section{Subalgebras of $\operatorname{sl}(2, \mathrm{C})$}

Our aim will be to study points in $\Gamma$ at which the coordinates $\left(T^{0}, T^{1}\right)$ become degenerate. The key to our consideration is the holonomy group $H$ of $A$. Since $H$ can be a priori an arbitrary subgroup of $S L(2, \mathbb{C})$, we first list all Lie subalgebras of $\operatorname{sl}(2, \mathbb{C})$.

In order to specify a basis in $\operatorname{sl}(2, \mathbb{C})$ let us fix a normalized basis in $V$, i.e. two spinors $o, \iota \in V$ with

$$
o^{A} \iota^{B} \varepsilon_{A B}=1 \text {. }
$$

Then define $\tau_{1}, \tau_{2}, \tau_{3} \in \operatorname{sl}(2, \mathbb{C})$ :

$$
\begin{aligned}
\tau_{1}{ }_{B}^{A} & :=i\left(o^{A} o_{B}-\iota^{A} \iota_{B}\right), \\
\tau_{2}{ }_{B}{ }_{B} & :=\left(o^{A} o_{B}+\iota^{A} \iota_{B}\right), \\
\tau_{3}{ }^{A}{ }_{B} & :=i\left(o^{A} \iota_{B}+\iota^{A} o_{B}\right) .
\end{aligned}
$$

The following linear combinations of $\tau_{1}$ and $\tau_{2}$ and their commutation relations will also be useful,

$$
\begin{gathered}
\tau_{+}:=\tau_{1}+i \tau_{2}, \quad \tau_{-}:=\tau_{1}-i \tau_{2}, \\
\tau_{ \pm} \tau_{3}= \pm i \tau_{ \pm}=-\tau_{3} \tau_{ \pm} \\
\tau_{+} \tau_{-}=-2(1+i) \tau_{3}, \quad \tau_{-} \tau_{+}=-2(1-i) \tau_{3} .
\end{gathered}
$$


We treat ' $\operatorname{sl}(2, \mathbb{C})$ as a 6-real-dimensional algebra spanned by generators $\left(\tau_{1}, \tau_{2}, \tau_{3}\right.$, $\left.i \tau_{1}, i \tau_{2}, i \tau_{3}\right)$. Every Lie subalgebra $\mathscr{A} \subset \operatorname{sl}(2, \mathbb{C})$ is equivalent $\left(\right.$ via $\left.a^{-1} \mathscr{A} a \equiv \mathscr{C}\right)$ to one of the subalgebras listed below consisting of real combinations of the following generators:

(a)

(b)

(c)

(d)

(e)

(f)

(g)

(h)

(i)

(j)

(o)

$$
\begin{gathered}
\left(\tau_{1}, \tau_{2}, \tau_{3}, i \tau_{1}, i \tau_{2}, i \tau_{3}\right), \\
\left(\tau_{1}, \tau_{2}, \tau_{3}\right), \\
\left(\tau_{1}, i \tau_{2}, i \tau_{3}\right), \\
\left(\tau_{+}, i \tau_{+}, \tau_{3}, i \tau_{3}\right), \\
\left(\tau_{+}, i \tau_{+}, e^{i \phi} \tau_{3}\right), \\
\left(\tau_{+}, i \tau_{3}\right), \\
\left(\tau_{3}, i \tau_{3}\right) \\
\left(\tau_{+}, i \tau_{+}\right) \\
\left(e^{i \phi} \tau_{3}\right) \\
\left(\tau_{+}\right)
\end{gathered}
$$

(0) .

(Note that a transformation $o \rightarrow \iota, \iota \rightarrow-o$ carries $\tau_{+} \rightarrow \tau_{-}$and $\tau_{3} \rightarrow-\tau_{3}$.) The subalgebra of the type (3.5d) plays a role in further considerations and we denote it by $\mathscr{A}(+, 3)$. Note that each $\mathscr{A}$ of one of the types $(3.5 \mathrm{~d})-(3.50)$ is included in $\mathscr{b}(+, 3)$.

Given a real algebra $\mathscr{A}_{0}$ spanned by generators $V_{i}$, i.e.

$$
\mathscr{b}=\left\{a^{i} V_{i} \mid a^{i} \in \mathbb{R}\right\},
$$

we denote by $\mathscr{A}_{\mathbf{C}}$ the complexification of $\mathscr{A}_{\text {, }}$, i.e.

$$
\mathscr{C}_{\mathbf{C}}=\left\{c^{i} V_{i} \mid c^{i} \in \mathbb{C}\right\}
$$

The list (3.5) leads to the following classification of the complexifications of subalgebras $\mathscr{A}$ of $\operatorname{sl}(2, \mathbb{C})$,

$$
\begin{aligned}
\mathscr{C} & =\operatorname{sl}(2, \mathbb{C}), \\
\mathscr{C}(+, 3) & =\left\{a \tau_{3}+b \tau_{+} \mid a, b \in \mathbb{C}\right\}, \\
\mathscr{C}(+) & =\left\{b \tau_{+} \mid b \in \mathbb{C}\right\}, \\
\mathscr{A}(3) & =\left\{a \tau_{3} \mid a \in \mathbb{C}\right\}, \\
\mathscr{A}(0) & =\{0\} .
\end{aligned}
$$

\section{Nondegenerate Points}

Consider closed loops $\{\gamma\}$ which begin and end in a fixed point $x \in \Sigma$. Given a loop $\gamma$ the corresponding holonomy element $h_{\gamma}$ may be written as

$$
h_{\gamma}=\cos \left|n_{\gamma}\right| I+\frac{n_{\gamma}}{\left|n_{\gamma}\right|}\left(1-\cos ^{2}\left|n_{\gamma}\right|\right)^{1 / 2}
$$


where $I$ denotes the unit matrix $\operatorname{diag}(1,1)$ and

$$
\begin{gathered}
n_{\gamma}=n_{\gamma}^{i} \tau_{i} \in \operatorname{sl}(2, \mathbb{C}), \\
\left.\left|n_{\gamma}\right|:=\left(\left(n_{\gamma}^{1}\right)^{2}+\left(n_{\gamma}^{2}\right)^{2}+\left(n_{\gamma}^{3}\right)^{2}\right)\right)^{1 / 2}
\end{gathered}
$$

with $n_{\gamma}^{i}$ being complex numbers. (Given a complex number $z$ as $z=\exp (2 i \phi)|z|$, where $\phi \in[0, \pi]$; then $\sqrt{z}=\exp (i \phi) \sqrt{|z|}$.) If $\left|n_{\gamma}\right|=0$ then (4.1) holds with

$$
\frac{\left(1-\cos ^{2}\left|n_{\gamma}\right|\right)^{1 / 2}}{\left|n_{\gamma}\right|} \rightarrow 1 \text {. }
$$

By (2.7),

$$
T_{\gamma}=2 \cos \left|n_{\gamma}\right| \text {. }
$$

Given composition $\gamma \circ \delta$ of two loops $\gamma$ and $\delta$

$$
h_{\gamma \circ \delta}=h_{\gamma} h_{\delta}
$$

and in the consequence

$$
T_{\gamma \circ \delta}=\frac{1}{2}\left[T_{\gamma} T_{\delta}-\left[\left(4-T_{\gamma}^{2}\right)\left(4-T_{\delta}^{2}\right)\right]^{1 / 2} \frac{n_{\gamma}^{i} n_{\delta}^{i}}{\left|n_{\gamma}\right|\left|n_{\delta}\right|}\right] .
$$

Hence, if $\left|n_{\kappa}\right|=0(\kappa=\gamma$ or $\delta)$ then the corresponding factor

$$
\frac{\left(4-T_{\kappa}^{2}\right)^{1 / 2}}{\left|n_{\kappa}\right|} \rightarrow 2
$$

Statement 4.1. If the complexification of the holonomy algebra of a connection $A$ is $\operatorname{sl}(2, \mathbb{C})$, and $A^{\prime}$ is a connection such that

$$
T_{\gamma}(A)=T_{\gamma}\left(A^{\prime}\right)
$$

holds for all the closed loops $\gamma$ with the starting point $x$, then $A$ is gauge equivalent to $A^{\prime}$.

Proof. The idea of the proof comes from Samuel [11]. Using $T^{0}$-functionals we will recover the holonomy at the point $x$ in a certain gauge fixed in $x$.

We can find three loops $\alpha, \beta$, and $\gamma$ such that

$$
\begin{gathered}
\left|n_{\alpha}\right|,\left|n_{\beta}\right|,\left|n_{\gamma}\right| \neq 0, \\
n_{\alpha}^{i} n_{\beta}^{i}=n_{\alpha}^{i} n_{\gamma}^{i}=n_{\beta}^{i} n_{\gamma}^{i}=0 .
\end{gathered}
$$

(It can be done since the holonomy algebra is spanned by generators (3.5a), (3.5b) or (3.5c).) The above properties of $n_{\alpha}, n_{\beta}$, and $n_{\gamma}$ can be found by using of the values of $T^{0}$ corresponding to the loops $\alpha, \beta, \gamma$ and to their superpositions $\alpha \circ \beta, \ldots$ Fix a gauge in the point $x$ by the requirement that

$$
\frac{n_{\alpha}}{\left|n_{\alpha}\right|}=\tau_{1}, \quad \frac{n_{\beta}}{\left|n_{\beta}\right|}=\tau_{2}, \quad \frac{n_{\gamma}}{\left|n_{\gamma}\right|}=\tau_{3} .
$$

(It may happen that we have to change names of the loops by $\alpha \leftrightarrow \beta$ - the proper order can be found by using of $T_{\alpha \circ \beta}^{a}, \ldots$ which involve the information about $\varepsilon_{i j k} n^{i} n^{k}$.) Given any loop $\delta$ we can find $h_{\delta}$ as follows: from $T_{\delta}, T_{\delta \circ \alpha}, T_{\delta \circ \beta}, T_{\delta \circ \gamma}$ and from Eq. (4.7) solved with respect to $n_{\delta}^{i} /\left|n_{\delta}\right|$ [see also (4.1)]. 
Since we obtain $h_{\delta}$ for each $\delta$ at $x$, we can find the connection 1-form $A$ on $\Sigma$ determined up to gauge transformations which concludes the proof.

Having Statement 4.1 in hand we can prove the following:

Theorem 4.2. Given $[(A, \sigma)]$ and $\left[\left(A^{\prime}, \sigma^{\prime}\right)\right]$ if the complexification of holonomy algebra of $A$ is $\operatorname{sl}(2, \mathbb{C})$, and

$$
T_{\gamma}(A)=T_{\gamma}\left(A^{\prime}\right), \quad T_{\gamma}^{a}(A, \sigma, y)=T_{\gamma}^{a}\left(A^{\prime}, \sigma^{\prime}, y\right)
$$

for all the closed loops $\gamma$ with the starting point $y$, and for all $y \in \Sigma$, then $[(A, \sigma)]$ is equal to $\left[\left(A^{\prime}, \sigma^{\prime}\right)\right]$.

Proof. From the equivalence of $T^{0}(A)$ and $T^{0}\left(A^{\prime}\right)$ [the first equation in (4.12)] and from the proof of Statement 4.1 we can see that we can find representatives $(A, \sigma)$ and $\left(A^{\prime}, \sigma^{\prime}\right)$ such that

$$
A^{\prime}=A
$$

Having chosen $A$ we can find $h_{\gamma}$ for any closed loop in $\Sigma$. The variables $\sigma^{a}$ can now be uniquely obtained from the $T^{1}$ functions. In fact, it suffices to use the equations

$$
T_{\kappa}^{a}=\left(1-\frac{T_{\kappa}^{2}}{4}\right)^{1 / 2} \frac{\operatorname{Tr}\left(n_{\kappa} \sigma^{a}\right)}{\left|n_{\kappa}\right|}
$$

at any $y \in \Sigma$ and for $\kappa=\alpha, \beta, \gamma$ such that the corresponding $n_{\kappa}$ [see (4.1)] are linearly (in the complex sense) independent. $\sigma^{\prime}$ is the solution to the same equations and thus is equal to $\sigma$.

\section{Degenerate Points of $\left(T^{0}, T^{1}\right)$}

We turn now to study cases, when the holonomy algebra $\mathscr{H}(A)$ of $A$ is of one of the remaining types, i.e. one of (3.5d)-(3.5o) or, in other words, when the complexification $\mathscr{H}_{\mathbf{C}}(A)$ of $\mathscr{H}(A)$ is a proper subalgebra of $\operatorname{sl}(2, \mathbb{C})$. Then

$$
\mathscr{H}(A) \subset \mathscr{B}(+, 3)
$$

[see (3.5d)]. Therefore there exists a gauge such that

$$
A=A^{3} \tau_{3}+A^{+} \tau_{+}
$$

The holonomy element associated to a loop $\gamma$ and a starting point $x \in \gamma$ is in general given by an ordered exponential, namely

$$
h_{\gamma}=P \exp \int_{\gamma} A
$$

(where the ordering $P$ depends on the choice of $x$ ). Since

$$
\left(\tau_{+}\right)^{2}=0
$$


and by (3.4) the series expansion of Eq. (5.3) contains only terms independent of $A^{+}$ or linear in $A^{+}$. Thus

$$
\begin{gathered}
h_{\gamma}=\cos \int_{\gamma} A^{3} I+\sin \int_{\gamma} A^{3} \tau_{3}+n_{\gamma}^{+} \tau_{+}, \\
n_{\gamma}^{+}=\int_{\gamma} J A^{+},
\end{gathered}
$$

where $J$ is a certain complicated function of $A_{a}^{3} \dot{\gamma}^{a}$. Our first observation is that $T_{\gamma}$ is independent of $A^{+}$, namely

$$
T_{\gamma}=2 \cos \theta_{\gamma}, \quad \theta_{\gamma}=\int_{\gamma} A^{3} .
$$

Therefore knowledge of $T_{\gamma}$ 's suffices only to reconstruct the $A^{3}$ component of $A$. However, $T_{\gamma}^{a}$ contains information about $A^{+}, \sigma^{3}$, and $\sigma^{-}$, where

$$
\sigma=\sigma^{3} \tau_{3}+\sigma^{+} \tau_{+}+\sigma^{-} \tau_{-} .
$$

Consider first a connection $A$ such that neither $A^{3}$ nor $A^{+}$can be gauge transformed to zero. That is, the holonomy algebra is of one of the types (3.5d)-(3.5f) thus the complexification $\mathscr{H}_{\mathbf{C}}(A)$ of $\mathscr{H}(A)$ is

$$
\mathscr{H}_{\mathrm{C}}(A)=\mathscr{A}(+, 3) .
$$

Suppose that in some point $x \in \Sigma$ the vector field

$$
\sigma^{a-}(x) \frac{\partial}{\partial x^{a}} \neq 0
$$

and consider loops that begin and end at $x$. Take two loops $\alpha$ and $\beta$ such that

$$
\begin{gathered}
h_{\alpha}=\cos \theta_{\alpha} I+\sin \theta_{\alpha} \tau_{3}+n_{\alpha}^{+} \tau_{+}, \\
h_{\beta}=1+n_{\beta}^{+} \tau_{+}, \\
\sin \theta_{\alpha} \neq 0 \neq n_{\beta}^{+} .
\end{gathered}
$$

We are free to perform a gauge transformation $a: \Sigma \rightarrow S L(2, \mathbb{C})$ which is compatible with (5.2), i.e.

$$
\begin{gathered}
a^{A}{ }_{B} \iota^{B}=B \iota^{A}+C o^{A}, \\
a^{A}{ }_{B} o^{B}=\frac{1}{B} o^{A},
\end{gathered}
$$

$B$ and $C$ being complex functions. By a suitable choice of $B$ and $C$ we carry $h_{\alpha}, h_{\beta}$ in (5.11) and (5.12) into

$$
\begin{gathered}
h_{\alpha}=\cos \theta_{\alpha} I+\tau_{3}\left(1-\cos ^{2} \theta_{\alpha}\right)^{1 / 2}, \\
h_{\beta}=1+\tau_{+} .
\end{gathered}
$$

Values of $\sigma^{a 3}(x)$ and $\sigma^{a-}(x)$ in this gauge are given by

$$
\begin{gathered}
T_{\alpha}^{a}=-\left[4-\left(T_{\alpha}\right)^{2}\right]^{1 / 2} \sigma^{a 3}(x), \\
T_{\beta}^{a}=-4 \sigma^{a-}(x) .
\end{gathered}
$$

The component $\sigma^{a+}$ does not appear in $\left(T^{0}, T^{1}\right)$. 
Now, let $h_{\gamma}$ be an arbitrary element of the holonomy group of $A$ at $x$,

$$
h_{\gamma}=\frac{1}{2} T_{\gamma} \pm \tau_{3}\left[1-\frac{\left(T_{\gamma}\right)^{2}}{4}\right]^{1 / 2}+n_{\gamma}^{+} \tau_{+} .
$$

We must still determine $n_{\gamma}^{+}$and the correct sign of the $\tau_{3}$. The sign can be specified by $T_{\gamma \circ \alpha}, T_{\alpha}$, and $T_{\gamma}$ using Eq. (4.7) [see also (4.8)]. To find $n_{\gamma}^{+}$we consider the quantity $T_{\gamma}^{a}$. Applying the equation

$$
T_{\gamma}^{a}(x)=-\left[4-\left(T_{\gamma}\right)^{2}\right] \sigma^{a 3}(x)-4 n_{\gamma}^{+} \sigma^{a-}(x),
$$

we express $n_{\gamma}^{+}$by $T_{\gamma}^{a}(x), T_{\gamma}, \sigma^{a 3}(x)$, and $\sigma^{a-}(x)$. Thus $h_{\gamma}$ is completely determined for any loop $\gamma$ starting at $x$. This information enables us to reconstruct the connection $A$ determined up to residual gauge transformations. Using $A$, we can find $h_{\gamma}$ 's at each other point $y \in \Sigma$. Finally, we read $\sigma^{3}(y)$ and $\sigma^{-}(y)$ from $h_{\gamma}$ 's, $T_{\gamma}^{a}$ 's, and $T_{\gamma}$ 's.

Summarizing, given $\left(T^{0}, T^{1}\right)$ which by assumption correspond to a certain $[(A, \sigma)]$ such that $\mathscr{H}_{\mathrm{C}}=\mathscr{b}(+, 3)$ and $\sigma^{a-}(x) \neq 0$ in at least one point $x \in \Sigma$ we recovered $A=A^{3} \tau_{3}+A^{+} \tau_{+}$and $\sigma^{a-}, \sigma^{a 3}$ at each point $y \in \Sigma$. A component $\sigma^{a+}$ remains arbitrary. The question arises however, whether one can find another $\left(A^{\prime}, \sigma^{\prime}\right)$ also corresponding to the same $\left(T^{0}, T^{1}\right)$ but possessing holonomy algebra which is not included in $\mathscr{A}(+, 3)$ or $\sigma^{\prime}$ such that $\sigma^{\prime-}=0$ everywhere. The answer is no.

In fact, each $T_{\gamma}, T_{\delta}$, and $T_{\gamma \circ \delta}$ computed for $A=A^{3} \tau_{3}+A^{+} \tau_{+}$satisfy (4.7) with

$$
n_{\gamma}^{i} n_{\delta}^{i}= \pm\left|n_{\gamma}\right|\left|n_{\delta}\right|
$$

Thus, they can not be associated to any connection form $A^{\prime}$ of the type (3.5a), (3.5b) nor (3.5c). Also if $\sigma^{\prime-}(x) \equiv 0$ then $T_{\gamma}^{\prime}=2$ implies $T_{\gamma}^{\prime a}(x)=0$ which is not true in the case of the considered $\left(T^{0}, T^{1}\right)$.

The examination of the cases when the holonomy algebra is of one of the types (3.5g)-(3.5o) can be done in a similar manner as above. Before stating the results and classifying the degeneracies of $\left(T^{0}, T^{1}\right)$ in $\Gamma$ let us make our notation precise. Given $[(A, \sigma)] \in \Gamma$ denote by

$$
\operatorname{Deg}[A, \sigma]
$$

the set of $\left[\left(A^{\prime}, \sigma^{\prime}\right)\right] \in \Gamma$ such that $\left(T^{0}, T^{1}\right)$ take the same values as in $[(A, \sigma)]$, i.e.

$$
\begin{aligned}
T_{\gamma}\left(A^{\prime}\right) & =T_{\gamma}(A), \\
T_{\gamma}^{a}\left(A^{\prime}, \sigma^{\prime}, y\right) & =T_{\gamma}^{a}(A, \sigma, y),
\end{aligned}
$$

for any closed loop $\gamma$ and any $y \in \gamma$. Using this definition we can state the following theorem which gives the sought for classification.

Theorem 5.1. Suppose $[(A, \sigma)] \in \Gamma$ and

(i) the complexification of the holonomy algebra

$$
\mathscr{H}_{\mathbf{C}}(A)=\operatorname{sl}(2, \mathbb{C}),
$$

then $\operatorname{Deg}[A, \sigma]$ consists of only one point

$$
\operatorname{Deg}[A, \sigma]=\{[(A, \sigma)]\} ;
$$

$$
\mathscr{H}_{\mathbf{C}}(A)=\mathscr{b}(+, 3)
$$


and $\sigma^{-}(x) \neq 0$ at some $x \in \Sigma$, then

$$
\begin{aligned}
\operatorname{Deg}[A, \sigma]=\{ & {\left[\left(A^{\prime}, \sigma^{\prime}\right)\right] \in \Gamma \mid\left(A^{\prime+}, A^{\prime 3}, A^{\prime-}, \sigma^{\prime 3}, \sigma^{\prime-}\right) } \\
& \left.=\left(A^{+}, A^{3}, 0, \sigma^{3}, \sigma^{-}\right) \text {and } \sigma^{\prime+} \text { is arbitrary }\right\}
\end{aligned}
$$

(iii)

$$
\mathscr{H}_{\mathrm{C}}(A)=\mathscr{C}(+),
$$

and $\sigma^{-}(x) \neq 0$ at some $x \in \Sigma$, then

$$
\begin{aligned}
\operatorname{Deg}[A, \sigma]=\{ & {\left[\left(A^{\prime}, \sigma^{\prime}\right)\right] \in \Gamma \mid\left(A^{\prime+}, A^{\prime 3}, A^{\prime-}, \sigma^{\prime-}\right) } \\
& \left.=\left(A^{+}, 0,0, \sigma^{-}\right) \text {and } \sigma^{\prime+}, \sigma^{\prime 3} \text { are arbitrary }\right\}
\end{aligned}
$$

(iv) either

(a)

$$
\mathscr{H}_{\mathbf{C}}(A)=\mathscr{C}(+, 3)
$$

and $\sigma^{-}=0$ on $\Sigma$, or

(b)

$$
\mathscr{H}_{\mathbf{C}}(A)=\mathscr{b}(3)
$$

then

$$
\begin{aligned}
\operatorname{Deg}[A, \sigma]=\{ & {\left[\left(A^{\prime}, \sigma^{\prime}\right)\right] \in \Gamma \mid\left(A^{\prime 3}, A^{\prime-}, \sigma^{\prime 3}, \sigma^{\prime-}\right) } \\
& \left.=\left(A^{3}, 0, \sigma^{3}, 0\right), \text { and } A^{\prime+}, \sigma^{\prime+} \text { arbitrary }\right\} \\
& \cup\left\{\left[\left(A^{\prime}, \sigma^{\prime}\right)\right] \in \Gamma \mid\left(A^{\prime+}, A^{\prime 3}, A^{\prime-}, \sigma^{\prime 3}\right)\right. \\
& \left.=\left(0, A^{3}, 0, \sigma^{3}\right) \text { and } \sigma^{\prime+}, \sigma^{\prime-} \text { arbitrary }\right\} ;
\end{aligned}
$$

(v) either

(a)

$$
\mathscr{H}_{\mathbf{C}}(A)=\mathscr{b}(+)
$$

and $\sigma^{-}(x)=0$, or

(b) $A$ is flat, then

$$
\begin{aligned}
\operatorname{Deg}[A, \sigma]=\{ & {\left[\left(A^{\prime}, \sigma^{\prime}\right)\right] \in \Gamma ;\left(A^{\prime 3}, A^{\prime-}, \sigma^{\prime-}\right) } \\
& \left.=(0,0,0), \text { and } A^{\prime+}, \sigma^{\prime+}, \sigma^{\prime 3} \text { arbitrary }\right\} \\
& \left.\cup\left\{\left[\left(A^{\prime}, \sigma^{\prime}\right)\right] \in \Gamma ; A^{\prime}=0\right) \text { and } \sigma^{\prime} \text { arbitrary }\right\} .
\end{aligned}
$$

Remark 5.1. The above classification exhausts all possible choices of $[(A, \sigma)]$.

\section{Other Descriptions of Degenerate Points}

As we saw in the previous section, points at which $\left(T^{0}, T^{1}\right)$ take the same value constitute leaves in $\Gamma$ which are unions of continuous surfaces. The leaves of the same type set up a class and classes are listed in Theorem 5.1. As we see below, the classes of Theorem 5.1 can be defined in terms of $\left(T^{0}, T^{1}\right)$.

Statement 6.1. The set of points at which $\left(T^{0}, T^{1}\right)$ become degenerate as coordinates of $\Gamma$ is given by the condition

$$
\left[2 T_{\alpha \circ \beta}-T_{\alpha} T_{\beta}\right]^{2}-\left[4-\left(T_{\alpha}\right)^{2}\right]\left[4-\left(T_{\beta}\right)^{2}\right]=0
$$

where $\alpha, \beta$ are closed loops in $\Gamma$ starting from the same point. 
Statement 6.2. Classes (i-v) in the Theorem 5.1 can be defined in the following way: (1) Class (v) (i.e. Deg[0,0]) is given by

$$
T_{\alpha}=2 ; \quad T_{\alpha}^{a}(y)=0,
$$

for any closed loop $\alpha$ and any $y \in \alpha$;

(2) Class (iii) is given by

$$
T_{\alpha}=2, \quad T_{\beta}^{a}(x) \neq 0,
$$

for each loop $\alpha$ and for some loop $\beta$ at some point $x \in \beta$,

(3) Class (iv) is given by the conditions that the following variation vanishes at each $\alpha$ and $y \in \alpha$,

$$
\frac{\delta}{\delta \alpha}\left[\frac{T_{\alpha}^{a}(y)^{2}}{4-\left(T_{\alpha}\right)^{2}}\right]=0,
$$

and that there exist loops $\alpha$ such, that

$$
\left(T_{\alpha}\right)^{2} \neq 4
$$

[Equation (6.5) ensures that the expression in brackets in (6.4) has a well defined limit when $\left.\left|\left(T_{\alpha}\right)\right| \rightarrow 2\right]$.

(4) Class (ii) consists of the $[(A, \sigma)]$ which satisfy Eq. (6.1) but are not contained in (1), (2), nor (3) above.

(5) Class (i) consists of the $[(A, \sigma)]$ such, that Eq. (6.1) is not in satisfied in general.

Note, that Class (v) of the Theorem 5.1 is in fact the set of $[(A, \sigma)]$ which are indistinguishable from $[(0,0)]$ with respect to $\left(T^{0}, T^{1}\right)$. In Classes (i), (ii), (iii), and (iv) the leaves $\operatorname{Deg}[A, \sigma]$ are labeled by $\left(T^{0}, T^{1}\right)$ according to the remaining freedom.

The fact that the complexified holonomy algebra of $A$ is included in $\mathscr{A}(+, 3)$ has a nice geometrical meaning. Then there exists on $\Sigma$ a spinor field such that its direction is covariantly constant. In fact,

$$
D o^{A}=d o^{A}-A^{A}{ }_{B} o^{B}=i A^{3} o^{A}
$$

[by (3.2)]. The converse, is also true: if there exists a spinor field $\lambda^{A} \neq 0$ such that

$$
\lambda_{A} D \lambda^{A}=0, \quad \lambda \neq 0,
$$

then in the spinor basis $(o, \iota)=(\lambda, \iota)$

$$
A=A^{+} \tau_{+}+A^{3} \tau_{3}
$$

On the other hand, $\lambda$ is covariantly constant, i.e.

$$
D \lambda=0
$$

if and only if $\mathscr{H}_{\mathbf{C}}(A)=\mathscr{b}(+)$.

The above observation about the existence of a covariantly constant spinor direction and field respectively will be applied to find an intersection of the set of degenerate $[(A, \sigma)]$ with the real slice of the constraint surface. 


\section{Hamiltonian Evolution of Holonomy Algebras}

The constrained surface in the phase space $\Gamma$ is defined by the vanishing of the following functions

$$
\begin{gathered}
\mathscr{C}:=\operatorname{Tr} \sigma^{a} \sigma^{b} F_{a b} \\
\mathscr{C}_{b}:=\operatorname{Tr} \sigma^{a} F_{a b}, \\
\mathscr{G}^{i}:=D_{a} \sigma^{a i}
\end{gathered}
$$

where

$$
F:=d A+A \wedge A \text {. }
$$

The time evolution $(A(t), \sigma(t))$ corresponding to the Hamiltonian constraint $\mathscr{C}$ and lapse function $N$ has the form

$$
\begin{aligned}
& \frac{d}{d t} A_{a}(t)=\frac{i}{\sqrt{2}}\left[N \sigma^{b}(t), F_{a b}(t)\right], \\
& \frac{d}{d t} \sigma^{a}(t)=\frac{i}{\sqrt{2}} D_{b}\left[N \sigma^{b}(t), \sigma^{a}(t)\right] .
\end{aligned}
$$

In other words curves $(A(t), \sigma(t))$ are required to be integral lines of the vector field

$$
X=\frac{1}{\sqrt{2}}\left[N \sigma^{b}, F_{a b}\right]^{\imath} \frac{\delta}{\delta A_{a}^{i}}+\frac{i}{\sqrt{2}} D_{b}\left[N \sigma^{b}, \sigma^{a}\right]^{i} \frac{\delta}{\delta \sigma^{a i}} .
$$

The $\sigma^{i}$ are three vector densities in $\Sigma$ of the weight 1 taking values in $\mathbb{C}$. In the next theorem we assume that $\left(\sigma^{i}\right)$ are linearly independent in the complex sense, i.e. that $a_{i} \sigma^{i}=0$ implies $a_{i}=0$ for any complex numbers $a_{\imath}$. This section is devoted to the proof of the following.

Theorem 7.1. Suppose $(A, \sigma)$ represents a point of the constraint surface for complex gravity and complex vectors $\left(\sigma^{i}\right), i=1,2,3$ are linearly independent in each point of $\Sigma$; then the Hamiltonian evolution $(A(t), \sigma(t))$ such that $(A(0), \sigma(0))=(A, \sigma)$ preserves the complexification $\mathscr{H}_{\mathrm{C}}(t)$ of the holonomy algebra of $A(t)$.

Proof. It suffices to show that the time evolution does not carry $\mathscr{H}_{\mathbf{C}}(t)$ out of the holonomy algebra $\mathscr{H}_{\mathrm{C}}$ of $A$,

$$
\mathscr{H}_{\mathbf{C}}(t) \subset \mathscr{H}_{\mathbf{C}}(0)
$$

Then we note, that the same is true when we change $t$ into $-t$ and $N$ into $-N$. Thus from (7.8) it follows that

$$
\mathscr{H}_{\mathbf{C}}(0) \subset \mathscr{H}_{\mathbf{C}}(t) \text {. }
$$

All the complex subalgebras of $\operatorname{sl}(2, \mathbb{C})$ are listed in (3.8). Let us first consider the three simpler cases. Namely, if $\mathscr{H}(0)=\{0\}$ then $F=0$ and by (7.5)

$$
\frac{d}{d t} A(t)=0 \text {. }
$$

On the other hand, if $\mathscr{H}_{\mathbf{C}}(0)=\operatorname{sl}(2, \mathbb{C})$, then it is obvious from $(7.5)$ that $\mathscr{H}_{\mathbf{C}}(t) \subset$ $\mathscr{H}_{\mathrm{C}}$.

Let $\mathscr{H}_{\mathrm{C}}(0)=\mathscr{C}_{(}(3)$. Then $A$ may be written as

$$
A=A^{3} \tau_{3} .
$$


The constraint equations $\mathscr{C}=0=\mathscr{C}_{a}$ read

$$
F_{a b}^{3} \sigma^{a-} \sigma^{b+}=0=F_{a b}^{3} \sigma^{a 3} .
$$

Thus, since $\sigma^{a+}, \sigma^{a-}$, and $\sigma^{a 3}$ are by assumption linearly independent,

$$
F=0 .
$$

But it is in contradiction with the assumption about the holonomy algebra, thus this case is excluded by the constraints.

Now let us turn to deal with a case when we are given $A$ such that $\mathscr{H}_{\mathbf{C}}(0)=\mathscr{A}(+)$, i.e. in some gauge

$$
A=A^{+} \tau_{+} .
$$

It is convenient to go to the space $\Gamma^{\prime}$ of pairs $(A, \sigma)$ (not divided by the gauge group) and introduce apart from the Hamiltonian vector field $X$ (7.7) a modified vector field

$$
Y=X-\frac{N}{\sqrt{2}} \mathscr{C}_{a} \frac{\delta}{\delta A_{a}^{3}},
$$

where $N$ is the same lapse function as in (7.7). The motivation to define this vector field is that $Y$ is tangent to the surface of $\left(A^{\prime}, \sigma^{\prime}\right)$ such, that

$$
A^{\prime 3}=0, \quad A^{\prime-}=0 .
$$

Thus, in other words, the condition (7.16) is preserved along the integral curves of $Y$. But integral curves of $Y$ agree with those of $X$ when we restrict ourselves to the constraint surface. Hence, it follows, that if $\left(A^{+} \tau_{+}, \sigma\right)$ satisfies the constraints then the Hamiltonian evolution preserves the form of $A(7.14)$ and the inclusion

$$
\mathscr{H}_{\mathbf{C}}(t) \subset \mathscr{H}_{\mathbf{C}}(0) .
$$

The last part of our proof is to study $\mathscr{H}_{\mathbf{C}}(t)$ when $\mathscr{H}_{\mathbf{C}}(0)=\mathscr{A}(+, 3)$. In this case the proof proceeds as above with a modified vector field in $\Gamma^{\prime}$ defined by

$$
Z=X+\sqrt{2} N\left[\left(\sigma_{a+}+\sigma_{a 3}\right) \sigma^{b-} \mathscr{C}_{b}-\frac{1}{4 i} \sigma_{a+} \mathscr{C}\right] \frac{\delta}{\delta A_{a}^{-}},
$$

where $\sigma_{a i} d x^{a}$ denotes the dual cobasis to $\sigma^{a i} \frac{\partial}{\partial x^{a}}$. Such a defined vector field $Z$ is tangent to the surface consisting of $\left(A^{\prime}, \sigma^{\prime}\right)$ such that

$$
A^{\prime-}=0 \text {. }
$$

On the other hand $Z=X$ on the constraint surface. Thus we can conclude that if $\left(A^{+} \tau_{+}+A^{3} \tau_{3}, \sigma\right)$ belongs to the constraint surface, then

$$
\mathscr{H}_{\mathbf{C}}(t) \subset \mathscr{H}_{\mathbf{C}}(0),
$$

which completes the proof.

Remark 7.1. The assumption that the vector fields $\left(\sigma^{a 1}, \sigma^{a 2}, \sigma^{a 3}\right)$ be linearly independent was not used in the proof above when the complexification of the holonomy algebra was

$$
\mathscr{H}_{\mathbf{C}}(0)=\mathscr{b}(+) .
$$

Remark 7.2. If $A$ is like a complex electromagnetic potential, i.e.

$$
A=A^{3} \tau_{3}
$$


then the constraints $\mathscr{C}=0=\mathscr{C}_{a}$ are not satisfied unless $A$ is flat or $\left(\sigma^{a 1}, \sigma^{a 2}, \sigma^{a 3}\right)$ are linearly dependent.

Statement 7.1. Theorem 7.1 does not hold if we drop the assumption that $\left(\sigma^{a 1}, \sigma^{a 2}\right.$, $\sigma^{a 3}$ ) define a complex tangent basis in each point of $\Sigma$.

This claim can be demonstrated by the following example.

Example 7.1. Let

$$
A=A^{3} \tau_{3}, \quad \sigma^{i} \tau_{i}=\sigma^{+} \tau_{+} .
$$

The scalar and diffeomorphism constraints are automatically satisfied, and the Gauss constraint reduces to

$$
\mathscr{G}^{+}=\sigma^{a+}-2 i A_{a}^{3} \sigma^{a+}=0 .
$$

Hence given an arbitrary $\sigma^{+}$, a connection $A^{3}$ is given, but not uniquely, by (7.20). For example, let $(u, x, y):=\left(x^{a}\right)$ be coordinates in $\Sigma$, and

$$
\begin{gathered}
\sigma=\sigma^{a+} \tau_{+} \frac{\partial}{\partial x^{a}}=\tau_{+} \frac{\partial}{\partial u} \\
A=(a d x+b d y) \tau^{3} .
\end{gathered}
$$

Suppose that Hamiltonian evolution of the above $(A, \sigma)$ leaves $\mathscr{H}_{\mathbf{C}}$ of $A$ invariant. Then the consistency conditions at the initial point of time are that $\tau_{+}$and $\tau_{-}$components of $\frac{d}{d t} A(t)$ vanish up to a gauge transformation. More precisely, we expect, that given a lapse funtion $N$ in (7.5) there exists an $\operatorname{sl}(2, \mathbb{C})$ field $\Lambda$ (generating a gauge transformation) such that

$$
\frac{i}{\sqrt{2}}\left[N \sigma^{b}, F_{a b}\right]^{ \pm}=D \Lambda^{ \pm}
$$

where ${ }^{+}$and ${ }^{-}$denotes $\tau_{+}$and $\tau_{-}$component respectively. Now, in particular, let us set $N=1$ and substitute $A=(d x+u d y) \tau_{3}$, and $\sigma$ of the form (7.21) into (7.23). We get for the $\tau_{+}$component

$$
\begin{gathered}
\frac{\partial}{\partial x} \Lambda^{+}=2 i \Lambda^{+} \\
\frac{\partial}{\partial y} \Lambda^{+}=2 i u \Lambda^{+}+\sqrt{2}
\end{gathered}
$$

which leads to a contradiction, namely

$$
\frac{\partial^{2}}{\partial x \partial y} \Lambda^{+}-\frac{\partial^{2}}{\partial y \partial x} \Lambda^{+}=-2 i \sqrt{2} \text {. }
$$

\section{Intersection of the Surface of Degenerate Points of $\left(T^{0}, T^{\mathbf{1}}\right)$ with the Surface of Real Vacuum gravitational Fields}

Up to now we have been studying the complexified gravity. Here we shall find the intersection between the real slice of the constraint surface and the surface on which $\left(T^{0}, T^{1}\right)$ coordinates in $\Gamma$ become degenerate. In order to do it, we extend a 3dimensional configuration $(\Sigma, A, \sigma)$ - by using the time evolution $(A, \sigma)(t)$ provided 
that the initial $(A, \sigma)$ satisfies constraints - to a 4-dimensional space-time $\Sigma \times \mathbb{R}$ endowed with the corresponding spinor structure ${ }^{4} \sigma_{\mu A B^{\prime}}$ and self dual spinor connection ${ }^{4} A_{\mu A B}$.

First we make a few observations about relations between $(A, \sigma)$ and $\left({ }^{4} A,{ }^{4} \sigma\right)$ before imposing the reality conditions.

Statement 8.1. If $(A, \sigma)$ satisfies the assumptions of Theorem 7.1 [see paragraph following Eq. (7.7)] then the complexified holonomy algebras ${ }^{4} \mathscr{H}_{\mathrm{C}}$ and $\mathscr{H}_{\mathrm{C}}$ of the connections ${ }^{4} A$ and $A$ respectively are equal to each other.

Proof. To construct ${ }^{4} A$, we immerse $\Sigma$ into $\Sigma \times \mathbb{R}$ and regard the time coordinate $t$ as the fourth coordinate completing coordinates $\left(x^{a}\right)$ in $\Sigma$ to a coordinate system in $\Sigma \times \mathbb{R}$. If the time evolution is given by $(7.5,7.6)$ then the spinor connection 1 -form ${ }^{4} A$ is defined as

$$
{ }^{4} A=A_{a}(t) d x^{a} .
$$

In the proof of Theorem 7.1 we have shown that $A(t)$ takes values in $\mathscr{H}_{\mathbf{C}}$. We wish to stress here that the essential reason why Statement 8.1 holds, is that we did not use in the proof of Theorem 7.1 any extra gauge term $D \Lambda(\Lambda$ being an $\operatorname{sl}(2, \mathbb{C})$ field $)$ to make

$$
\mathscr{H}_{\mathbf{C}}(t)=\mathscr{H}_{\mathbf{C}} \text {. }
$$

Otherwise, the $A_{t} d t$ term should have to appear in (8.1) which would have spoiled our argument.

Let us go back now, to the results of Sect.2. According to Theorem 7.1 and Statement 8.1 degenerate points $(A, \sigma)$ of $\left(T^{0}, T^{1}\right)$ correspond to connection ${ }^{4} A$ of the complexified holonomy algebra belonging to one of the following cases:

$$
\begin{gathered}
{ }^{4} \mathscr{H}_{\mathbf{C}}=\{0\}, \\
{ }^{4} \mathscr{H}_{\mathbf{C}}=\mathscr{b}_{(+)},
\end{gathered}
$$

or

$$
{ }^{4} \mathscr{H}_{\mathbf{C}}=\mathscr{B}(+, 3) .
$$

We can easily establish the Petrov type of the curvature

$$
{ }^{4} F=d^{4} A+{ }^{4} A \wedge{ }^{4} A .
$$

In the case $(8.3 a){ }^{4} F=0$. On the other hand in the case $(8.3 b)$

$$
{ }^{4} F^{A}{ }_{B}=2 i^{4} F^{+} o^{A} o_{B}
$$

[see (3.2)]. It follows that

$$
{ }^{4} F^{A}{ }_{B} o^{B}=0
$$

thus $o^{A}$ is a fourfold spinor of the Weyl spinor (Petrov type $N$ ).

In the case (8.3c) we have

$$
{ }^{4} F^{A}{ }_{B} o^{B} o_{A}=0 \neq{ }^{4} F^{A}{ }_{B} o^{B} .
$$

Hence $o^{A}$ is a triple principal spinor of the Weyl spinor (Petrov type III).

We can also repeat the arguments of Sect. 6 to claim that ${ }^{4} \mathscr{H}_{\mathrm{C}}$ is of the type (8.3b) if and only if there exists in $\Sigma \times \mathbb{R}$ a covariantly constant (with respect to ${ }^{4} A$ ) nontrivial spinor field $o$,

$$
{ }^{4} D o^{A}=0,
$$


and ${ }^{4} \mathscr{H}_{\mathbf{C}}$ is of the type (8.3c) if and only if there exists a covariantly constant spinor direction $o^{A}$, i.e.

$$
o_{A}^{4} D o^{A}=0 \text {. }
$$

Finally, let us focus our attention on $\left({ }^{4} A,{ }^{4} \sigma\right)$ corresponding to a real gravitational field. In terms of initial data $(\Sigma, A, \sigma)$ the reality of the metric on $\Sigma$ is expressed by

$$
\operatorname{Im} \operatorname{Tr} \sigma^{a} \sigma^{b}=0,
$$

and the reality conditions on the connection $A$ are given by the propagation of (8.10)

$$
\operatorname{Re} \operatorname{Tr}\left(\sigma^{a} \sigma^{c} D_{c} \sigma^{b}+\sigma^{b} \sigma^{c} D_{c} \sigma^{a}\right)=0 .
$$

Below we shall refer to these conditions as the "3-metric reality condition," and the "evolution reality condition," respectively. However, in the spirit of this section the reality conditions are that $\left({ }^{4} A,{ }^{4} \sigma\right)$ are the self dual part of the spinorial connection and the spinorial structure of a real Riemann geometry with metric tensor ${ }^{4} g$ of Lorentz signature (because of the constraints ${ }^{4} g$ is a solution to the vacuum Einstein equations).

In the real case, Eq. (8.8) means that there exists a (real) null vector $k$, namely

$$
k^{\mu} \equiv o^{A} \bar{o}^{A^{\prime}}
$$

which is covariantly constant:

$$
{ }^{4} D k^{\mu}=0
$$

(we extend the meaning of ${ }^{4} D$ to the Riemannian connection related to ${ }^{4} A$ and ${ }^{4} \sigma$ ). The vacuum solutions to the Einstein equations admitting a covariantly constant vector field are called p.p. waves [15]. Locally the metric tensor can be expressed by suitably chosen coordinates $(z=x+i y, \bar{z}=x-i y, u, r)$ as

$$
\begin{gathered}
g=2 d z d \bar{z}-2 d u(d r+H d u), \\
H=\operatorname{Re} h(z, u),
\end{gathered}
$$

where $h(z, u)$ is an arbitrary function holomorphic in $z$.

The spinor transformation algebra $\operatorname{sl}(2, \mathbb{C})$ is now regarded as $o(3,1)$ algebra of Lorentz transformations, while the holonomy algebra $(8.3 \mathrm{c})$ is identified with the algebra generating null rotations which preserve the distinguished null direction $k$. It is consistent with (8.9) which in the real case implies

$$
{ }^{4} D k^{\mu} \sim k^{\mu} .
$$

The class of vacuum gravitational fields which possess a holonomy group consisting of null rotations was studied by Goldberg and Kerr $[13,14]$. The Einstein equations were completely solved for that case, and a metric tensor was derived in the following form:

$$
g=2 d z d \bar{z}-2 d u(d r+W d z+\bar{W} d \bar{z}+H d u)
$$

where

$$
\begin{gathered}
W=\overline{f(z, u)}, \\
H=\frac{1}{2}\left(W_{\bar{z}}+\bar{W}_{z}\right) r+H^{0}, \\
H^{0}=\operatorname{Re}\left[\left(W W_{\bar{z}}+W_{u}\right) z+h(z, u)\right],
\end{gathered}
$$

and $f(z, u), h(z, u)$ are arbitrary functions holomorphic in $z$. 
In both cases (8.14) and (8.17)

$$
o^{A} \bar{o}^{A^{\prime}} \equiv \frac{\partial}{\partial r}
$$

and the holonomy algebras (as sets) of ${ }^{4} A$ are equal to their complexifications.

\section{9. $\left(T^{0}, T^{1}\right)$ Restricted to the real Slice $\Gamma_{R}$ of the Phase Space $\Gamma$}

In terms of the phase space variables $A, \sigma$, the real slice $\Gamma_{\mathbb{R}} \subset \Gamma$ consists of points which satisfy the reality conditions $(8.10)$ and (8.11).

We will study in this section $\left(T^{0}, T^{1}\right)$ restricted to $\Gamma_{\mathbb{R}}$. For $[(A, \sigma)] \in \Gamma_{\mathbb{R}}$, if $\left(T^{0}, T^{1}\right)$ is invertible at $[(A, \sigma)]$ in $\Gamma$ then it is also invertible at this point in $\Gamma_{\mathbb{R}}$. Thus again, degeneracy of $\left(T^{0}, T^{1}\right)$ can appear only when the complexified holonomy Lie algebra $\mathscr{H}_{\mathrm{C}}$ of $A$ is equivalent to one of the following: $\mathscr{A}_{(}(+, 3), \mathscr{A}_{(}(+), \mathscr{C}_{(}(3)$ or $\mathscr{A}(0)$. The degeneracy is described by the set

$$
\operatorname{Deg}_{\mathbb{R}}[A, \sigma]:=\operatorname{Deg}[A, \sigma] \cap \Gamma_{\mathbb{R}} .
$$

We shall divide our considerations into two parts. First we shall consider the restrictions on $\Gamma_{\mathbb{R}}$ imposed by the 3-metric reality condition (8.10) and only after that consider the further restrictions due to the evolution reality condition (8.11).

Henceforth, we will assume that the spinorial triad $\sigma^{i}\left(\sigma=: \sigma^{i} \tau_{i}\right)$ is $\mathbb{C}$-linearlyindependent and that the signature of $\operatorname{Tr}\left(\sigma^{a} \sigma^{b}\right)$ is $(+++)$. Then, the 3-metric reality condition (8.10) implies that there exists a gauge such that

$$
\sigma^{+}=\overline{\sigma^{-}}, \quad \sigma^{3}=\overline{\sigma^{3}} \text {. }
$$

\section{a) Degeneracy Restricted only by the 3-Metric Reality Condition (8.10)}

From now on, our gauge freedom is broken down to the subgroup of real gauge transformations which preserve Eq. (9.2), i.e. to $S U(2) \subset S L(2, \mathbb{C})$. It turns out that if $\mathscr{H}_{\mathrm{C}}(A)$ is of the type $\mathscr{C}(+, 3), \mathscr{b}(+)$ or $\mathscr{b}(0)$ up to an $S L(2, \mathbb{C})$ gauge transformation, then there exists an $S U(2)$ gauge transformation which carries $\mathscr{H}_{\mathrm{C}}(A)$ respectively into $\mathscr{A}(+, 3), \mathscr{b}(+)$ or $\mathscr{b}(0)$. On the other hand $\mathscr{H}_{\mathbf{C}}(A)$ is of the type $\mathscr{f}(3)$, then using $S U(2)$ gauge transformations we can establish only

$$
\begin{gathered}
\mathscr{H}_{\mathbf{C}}(A)=\left\{a \tau_{3}(\phi) ; a \in \mathbb{C}\right\}, \\
\tau_{3}(\phi)=\exp \left(-\phi \tau_{+}\right) \tau_{3} \exp \left(\phi \tau_{+}\right),
\end{gathered}
$$

where $\phi$ is a real function in $\Sigma$. Furthermore, given a connection $A$, such that the holonomy algebra $\mathscr{H}(A)$ is a proper subalgebra of $\operatorname{sl}(2, \mathbb{C}), A$ itself may not take values in that subalgebra. Of course, we can always find a (complex) gauge transformation such that $A$ does lie in $\mathscr{H}(A)$. However, here we are confined to real gauge transformations and we can write a connection $A$ with $\mathscr{H}_{\mathbf{C}}$ of the form $\mathscr{C}_{(}(+, 3)$, $\mathscr{A}(+), \mathscr{C}(3)$ or $\mathscr{C}(0)$ as respectively

$$
\begin{gathered}
A=A^{+} \tau_{+}+A^{3} \tau_{3}, \\
A=e^{-2 \varphi} a \tau_{+}+\mathrm{id} \varphi \tau_{3}, \\
A=A^{3} \tau_{3}(\phi)+d \phi \tau_{+}, \\
A=g^{-1} d g,
\end{gathered}
$$


$A^{+}, A^{3}, a$ being $\mathbb{C}$-1-forms, $\varphi$ and $\phi$ being real functions and with $S L(2, \mathbb{C})$-valued function $g$. Denote by $\operatorname{Deg}_{1}[A, \sigma] \subset \operatorname{Deg}[A, \sigma][$ see $(5.23,5.24)$ and above] the set of all the elements which satisfy the reality condition $(9.2)$. Given $[(A, \sigma)]$ we will find $\operatorname{Deg}_{1}[A, \sigma]$ in two steps. First we specify all real $\sigma^{\prime a}$ and mappings $\gamma \rightarrow h_{\gamma}^{\prime} \in$ $S L(2, \mathbb{C})\left(S U(2)\right.$-gauge inequivalent) such that $\left(\operatorname{Tr} h_{\gamma}^{\prime}, \operatorname{Tr} h_{\gamma}^{\prime} \sigma^{\prime a}\right)=\left(T^{0}, T^{1}\right)(A, \sigma)$. Then we find all $A^{\prime}$ which correspond to $h_{\gamma}^{\prime}$. Since two connections $A, A^{\prime}$ have the same holonomy $h_{\gamma}$ (for each loop $\gamma$ with a starting point $y \in \Sigma$ ) if and only if

$$
A^{\prime}=g^{-1} A g+g^{-1} d g,
$$

$g(y)$ commuting with all the elements of the holonomy group of $A$ at $y \in \Sigma$, each of the cases $(9.4 \mathrm{a}-9.4 \mathrm{~d})$ will be treated separately. We begin with the case $(9.4 \mathrm{c})$. A holonomy element is of the form

$$
h_{\gamma}=\cos \theta_{\gamma} I+\sin \theta_{\gamma} \tau_{3}(\phi) .
$$

The $T^{0}$ 's equivalence implies that

$$
h_{\gamma}^{\prime}=\cos \theta_{\gamma} I+\sin \theta_{\gamma} \tau_{3}\left(\phi^{\prime}\right) .
$$

Comparing $T_{\gamma}^{a}$ and $T_{\gamma}^{a^{\prime}}$ we find

$$
\operatorname{Tr} \sigma^{\prime} \tau_{3}\left(\phi^{\prime}\right)=\operatorname{Tr} \sigma \tau_{3}(\phi) .
$$

From this one condition we see that there is a large class of real $\sigma^{\prime}, \phi^{\prime}$ which have the same $T^{1}$. Holonomy (9.7) corresponds to any $A^{\prime}$ given by

$$
A^{\prime}=\left(A^{3}+\operatorname{id} \varphi\right) \tau_{3}\left(\phi^{\prime}\right)+d \phi^{\prime} t_{+},
$$

where $\varphi$ in is an arbitrary real function which doesn't change holonomy and represents an additional degree of freedom.

Turn now to the case $(9.4 \mathrm{~b})$. Here we have

$$
h_{\gamma}=1+n_{\gamma}^{+} \tau_{+} .
$$

Suppose, that $h_{\gamma}, \sigma$ and $h_{\gamma}^{\prime}, \sigma^{\prime}$ possess the same $\left(T^{0}, T^{1}\right)$. We get

$$
h_{\gamma}^{\prime}=1+n_{\gamma}^{\prime+} \tau_{+}, \quad n_{\gamma}^{+} \sigma^{a-}=n_{\gamma}^{\prime+} \sigma^{a-} .
$$

Hence, there exists such a function $k$ that

$$
n_{\gamma}^{\prime+}=\frac{1}{k} n_{\gamma}^{+}, \quad \sigma^{\prime-}=k \sigma^{-},
$$

for each $\gamma$. By real gauge transformations we can make $k$ real. Equation $\left(9.11^{\prime}\right)$ is equivalent to

$$
h_{\gamma}^{\prime}=\exp \left(-\frac{1}{2} \ln k \tau_{3}\right) h_{\gamma} \exp \left(\frac{1}{2} \ln k \tau_{3}\right),
$$

and such holonomy can be associated to any $A^{\prime}$ of the form

$$
A^{\prime}=\frac{e^{-2 \varphi}}{k}(a+d \lambda) \tau_{+}+i\left(d \varphi+\frac{1}{2} d \ln k\right) \tau_{3},
$$

where $\lambda$ is an arbitrary complex function creating an additional degree of freedom and $\varphi$ is as in (9.9). 
In the case (9.4a) the correspondence between the holonomy

$$
h_{\gamma}=\cos \theta_{\gamma} I+\tau_{3} \sin \theta_{\gamma}+n_{\gamma}^{+} \tau_{+}
$$

and the connection is unique. Suppose again that $(A, \sigma)$ and $\left(A^{\prime}, \sigma^{\prime}\right)$ have the same $\left(T^{0}, T^{1}\right)$. As in the case $(9.4 \mathrm{c}) \cos \theta_{\gamma}$ and $\sin \theta_{\gamma}$ are determined by $T^{0}$.

In order to connect $\sigma^{a-}$ with $\sigma^{a-}$, select a loop $\gamma$ such that

$$
h_{\gamma}=1+n_{\gamma}^{+} \tau_{+},
$$

(such $\gamma$ exists). Then via $T_{\gamma}=T_{\gamma}^{\prime}=2$,

$$
h_{\gamma}^{\prime}=1+n_{\gamma}^{\prime+} \tau_{+} .
$$

From $T_{\gamma}^{a}=\left(T^{\prime}\right)_{\gamma}^{a}$ we have

$$
n_{\gamma}^{\prime+} \sigma^{a-}=n_{\gamma}^{+} \sigma^{a-}
$$

It follows that

$$
\sigma^{a-}=k \sigma^{a-},
$$

$k$ being a function on $\Sigma$. Now, let us choose another loop $\beta$ such that

$$
\sin \theta_{\beta} \neq 0 \text {. }
$$

Then $T^{1}$ equivalence follows:

$$
\left(\sigma^{a 3}-\sigma^{\prime a 3}\right) \sin \theta_{\beta}+2 \sigma^{a-}\left(n_{\beta}^{\prime+} k-n_{\beta}^{+}\right)=0 .
$$

Note that since $\operatorname{Re} \sigma^{a-}$ and $\operatorname{Im} \sigma^{a-}$ are linearly independent vectors, it has to be

$$
\sigma^{\prime a 3}=\sigma^{a 3}, \quad n_{\beta}^{++}=\frac{1}{k} n_{\beta}^{+}
$$

for $\sigma^{a 3}$ is real. As in the case (9.4b) we claim that such a change of holonomy group is obtained by

$$
A^{\prime}=\frac{1}{k} A^{+} \tau_{+}+\left(A^{3}+\frac{i}{2} d \ln k\right) \tau_{3}
$$

$k$ being a real function on $\Sigma$ (we make it real by gauge transformations). Note that here, unlike in the case $(9.4 b, c)$, we have no freedom to change connection when $h_{\gamma}$ 's are fixed.

To summarize our considerations we have

Statement 9.1. If vectors $\sigma^{i}$ satisfy reality condition (9.2) and are linearly independent at each point of $S$, then for each type of the holonomy algebra $\mathscr{H}(A) \equiv \mathscr{b}(+, 3)$, $\mathscr{A}(+)$ or $\mathscr{A}(3) \operatorname{Deg}_{1}[A, \sigma]$ consists of all $\left[\left(A^{\prime}, \sigma^{\prime}\right)\right]$ with the following restrictions: (a) $\mathscr{H}(A) \equiv \mathscr{b}(+, 3)$ :

$$
A^{\prime}=\frac{1}{k} A^{+} \tau_{+}+\left(A^{3}+\frac{i}{2} d \ln k\right) \tau_{3} \quad \sigma^{\prime}=k\left(\sigma^{+} \tau_{+}+\sigma^{-} \tau_{-}\right)+\sigma^{3} \tau_{3}
$$

$k$ being a real function;

(b) $\mathscr{H}(A) \equiv \mathscr{A}(+)\left(A=e^{-2 \varphi} a \tau_{+}+\mathrm{id} \varphi \tau_{3}\right)$ :

$$
A^{\prime}=\frac{e^{-2 \varphi}}{k}(a+d \lambda) \tau_{+}+i\left(d \varphi+\frac{1}{2} d \ln k\right) \tau_{3}, \quad \sigma^{\prime}=k\left(\sigma^{+} \tau_{+}+\sigma^{-} \tau_{-}\right)+\sigma^{3} \tau_{3}
$$


$k$ and $\lambda$ being a real and a complex function respectively, and $\sigma^{\prime 3}$ being a real vector field;

(c) $\mathscr{H}(A) \equiv \mathscr{A}(3)\left(A=A^{3} \tau_{3}(\phi)+d \phi \tau_{+}\right.$[see (9.3)]:

$$
A^{\prime}=\left(A^{3}+\operatorname{id} \varphi\right) \tau_{3}\left(\phi^{\prime}\right)+d \phi^{\prime} \tau_{+}, \quad \operatorname{Tr} \sigma^{\prime} \tau_{3}\left(\phi^{\prime}\right)=\operatorname{Tr} \sigma \tau_{3}(\phi),
$$

$\phi^{\prime}$ and $\varphi$ being real functions, $\sigma^{\prime}$ being real [see (9.2)].

\section{(b) Degeneracy Restricted by the 3-Metric Reality Condition and the Evolution Reality Condition}

Thus far we restricted $\left(T^{0}, T^{1}\right)$ to $(A, \sigma)$ which satisfy only the first reality condition (8.10). The next step is to impose on $(A, \sigma)$ and on $\left(A^{\prime}, \sigma^{\prime}\right)$ the evolution reality condition (8.11). Suppose that $\sigma$ is real in the sense of $(9.2)$ and $\left(\sigma^{+}, \sigma^{3}, \sigma^{-}\right)$are linearly independent vectors in each point of $\Sigma$. Then the condition (8.11) imposed on $A=A^{+} \tau_{+}+A^{3} \tau_{3}$ and $\sigma$ reads (below $A_{j}^{i}:=A_{a}^{i} \sigma^{a j}, i, j=+, 3,-$ and $\sigma_{j}$ denotes the dual cobasis to $\sigma^{i}$ )

$$
\begin{gathered}
\operatorname{Re}\left(A_{-}^{+}\right)=\frac{1}{2 i} \sigma_{3}\left(\left[\sigma^{+}, \sigma^{-}\right]\right) \\
A_{3}^{+}+A_{+}^{3}+\overline{A_{-}^{3}}=i \sigma_{-}\left(\left[\sigma^{+}, \sigma^{-}\right]\right)-i \sigma_{3}\left(\left[\sigma^{+}, \sigma^{3}\right]\right) \\
A_{+}^{+}=\frac{i}{2} \sigma_{-}\left[\left(\sigma^{3}, \sigma^{+}\right)\right] \\
\operatorname{Re}\left(A_{3}^{3}+A_{-}^{+}\right)=\frac{i}{4} \sigma_{+}\left(\left[\sigma^{+}, \sigma^{3}\right]\right)-\frac{i}{4} \sigma_{-}\left(\left[\sigma^{-}, \sigma^{3}\right]\right) .
\end{gathered}
$$

Hence, the set $\operatorname{Deg}_{\mathbb{R}}[A, \sigma]$ consists of those points of $\operatorname{Deg}_{1}[A, \sigma]$ given - according to the holonomy type of $A$ - by Statement 9.1, which satisfy Eqs. (9.25). ( $\left.T^{0}, T^{1}\right)$ become degenerate in $[(A, \sigma)]$ if $\operatorname{Deg}_{\mathbb{R}}[A, \sigma]$ has more than one element.

We will find now a local expression for $[(A, \sigma)]$ at which the real degeneracy appears provided that neither $A^{+}$nor $A^{3}$ can be gauged to zero, that is in the case when $H_{\mathbf{C}}(A)$ is 4-dimensional. Then $\operatorname{Deg}_{1}[A, \sigma]$ is given by Statement 9.1(a). Suppose that $[(A, \sigma)],\left[\left(A^{\prime}, \sigma^{\prime}\right)\right] \in \operatorname{Deg}_{1}[A, \sigma]$ and that both of them satisfy $(9.25)$. Then $\left(A^{\prime}, \sigma^{\prime}\right)$ are given by Statement 9.1 (a) with $k$ being a real function in $\Sigma$ which is not identically equal to 1 (provided that $\left.\left[\left(A^{\prime}, \sigma^{\prime}\right)\right] \neq[(A, \sigma)]\right)$. In that region of $\Sigma$ on which

$$
k \neq 1 \text {, }
$$

Equation (9.25a) applied for $(A, \sigma)$ and for $\left(A^{\prime}, \sigma^{\prime}\right)$ is equivalent to

$$
\operatorname{Re}\left(A_{-}^{+}\right)=0=\sigma_{3}\left(\left[\sigma^{+}, \sigma^{-}\right)\right] .
$$

The substitution of $(A, \sigma)$ and $\left(A^{\prime}, \sigma^{\prime}\right)$ into (9.25b) implies

$$
A_{3}^{+}=\frac{1}{2 i} \sigma^{a+} \frac{\partial}{\partial x^{a}} \ln \left(k^{2}-1\right) .
$$

On the other hand Eqs. $(9.25 \mathrm{c}, \mathrm{d})$ are satisfied automatically by those $\left(A^{\prime}, \sigma^{\prime}\right)$ if $(A, \sigma)$ is their solution. A local expression for $(A, \sigma)$ which satisfies Eqs. (9.25), (9.27), and (9.28) with some $k$ can be found in an explicit form. Given $k$ Eq. (9.28) determines $A_{3}^{+}$. Then any other admissible $k^{\prime}$ is given by

$$
k^{2}=\left(k^{2}-1\right) b+1,
$$


where $b$ is a real function such that

$$
\sigma^{a+} \frac{\partial}{\partial x^{a}} b=0 .
$$

The geometrical interpretation of the right equation in (9.27) is that the vector fields $\operatorname{Re} \sigma^{+}, \operatorname{Im} \sigma^{+}$are surface forming. Let $u=$ const label leaves of the foliation. By the same reason for which each 2-dim metric tensor can be written in the form $d s^{2}=H^{2}\left(d^{2} x+d^{2} y\right)$ we can find such coordinates $(x, y, u)$ on $\Sigma$ that with respect to them

$$
\sigma^{+}=\alpha \frac{\partial}{\partial \bar{z}}, \quad \sigma^{-}=\alpha \frac{\partial}{\partial z}
$$

where $\alpha$ is a function (by an $S U(2)$ gauge transformation $\alpha$ has been made real) and

$$
z:=x+i y \text {. }
$$

To complete this construction, given $\sigma^{+}$and $\sigma^{-}$as in (9.31), an arbitrary real $\sigma^{3}$ and a real function $k$, we find $A_{3}^{+}$by $(9.28), \operatorname{Re} A_{-}^{+}$by $(9.27), A_{+}^{+}$by $(9.25 \mathrm{c}) \operatorname{Re} A_{3}^{3}$ from (9.25d) and $A_{+}^{3}+\overline{A_{-}^{3}}$ from (9.25b) while the remaining components of $A$ are arbitrary. Summarizing, given $[(A, \sigma)] \in \Gamma_{\mathbb{R}}$ there exists $\left[\left(A^{\prime}, \sigma^{\prime}\right)\right] \in \Gamma_{\mathbb{R}}$ of the same form as in Statement 9.1(a) if and only if there exists an open region $\Omega \subset \Sigma$ and a real function $k$ (globally defined in $\Sigma$ and smooth) such that

$$
k \equiv 1 \text { on } \quad \Sigma \backslash \Omega
$$

and $A, \sigma$ agree on $\Omega$ with the above construction, where $z, u$ are defined in some neighbourhood of each $y \in \Omega$. If

$$
\Omega=\Sigma
$$

above and the functions $z, u$ are defined globally, then it is clear that points $\left[\left(A^{\prime}, \sigma^{\prime}\right)\right] \in$ $\operatorname{Deg}_{\mathbb{R}}[A, \sigma]$ are labeled by functions $k^{\prime}$ of the form

$$
k^{\prime 2}=b(u)\left(k^{2}-1\right)+1,
$$

with an arbitrary real function $b$ of $u$.

\section{0. $\left(T^{0}, T^{1}\right)$ Coordinates Restricted to the Real Slice of the Constraint Surface}

In this section we try to answer the question posed by Rovelli and Smolin whether $\left(T^{0}, T^{1}\right)$ parametrize the slice of the constraint surface which corresponds to real solutions of the vacuum Einstein equations. The points of this slice at which $\left(T^{0}, T^{1}\right)$ become degenerate, while considered as coordinates in the whole phase space $\Gamma$ for complex gravity, correspond to the Goldberg-Kerr metric tensors (8.17-8.20). Sets of a constant value of $\left(T^{0}, T^{1}\right)$ restricted to the real constraint surface are denoted by $\operatorname{Deg}_{\mathbb{R c o n}}[A, \sigma]$; i.e.

$$
\operatorname{Deg}_{\mathbb{R} \text { con }}[A, \sigma]=\operatorname{Deg}_{\mathbb{R}}[A, \sigma] \cap \text { constraint surface } .
$$

Our first step will be to write the explicit expressions for $(A, \sigma)$ associated to these solutions. Each 3-dimensional and not null surface $\Sigma$ in a space-time (8.17) can be described (locally) by an equation

$$
r=\phi(z, \bar{z}, u)
$$


$\phi$ being a real function independent of $r$. The determinant of $q_{a b}(a, b=x, y, u)-$ the induced metric on $\Sigma$ - is proportional to

$$
\alpha^{2}:=-\left(W+\phi_{z}\right)\left(\bar{W}+\phi_{\bar{z}}\right)-H(z, \bar{z}, u, r=\phi)-\phi_{u} .
$$

Thus we consider functions $\phi(z, \bar{z}, u)$ in (10.1) such that

$$
\alpha^{2}>0 \text {. }
$$

We provide $\Sigma$ with $(A, \sigma)$ constructed from the metric tensor $g$ and from the Riemannian connection as described in [3]. We obtain with

$$
\begin{gathered}
Z:=W+\phi_{z} \\
\sigma=\frac{1}{2}\left[\tau^{+} \alpha \frac{\partial}{\partial \bar{z}}+\tau^{-} \alpha \frac{\partial}{\partial z}-\tau^{3}\left(\frac{\partial}{\partial u}+\bar{Z} \frac{\partial}{\partial z}+Z \frac{\partial}{\partial \bar{z}}\right)\right] \\
A^{+}=\frac{i}{2} \alpha_{\bar{z}} \sigma_{3}+\frac{i}{4} \bar{Z}_{\bar{z}} \sigma_{+}+\frac{i}{8}\left(\bar{Z}_{z}+Z_{\bar{z}}\right) \sigma_{-} \\
A^{3}=-\frac{i}{4} W_{\bar{z}} \sigma_{3}+\frac{i}{2} d(\ln \alpha)
\end{gathered}
$$

where

$$
\sigma_{3}:=-2 d u, \quad \sigma_{+}:=\frac{2}{\alpha}(d \bar{z}-Z d u)=: \overline{\sigma_{-}},
$$

and $W(\bar{z}, u), H(z, \bar{z}, u, r)$ are the same as in (8.17-8.20). It follows from (10.4d) that the complexified holonomy algebra of $A$ is of the type $\mathscr{b}(+, 3)$ if and only if

$$
W_{\bar{z} \bar{z}} \neq 0 \text {. }
$$

If conversely,

$$
W_{\bar{z} \bar{z}}=0,
$$

then $\mathscr{H}_{\mathbf{C}}$ is of the type $\mathscr{b}(+)$ and by a suitable choice of coordinates $(z, \bar{z}, u, r)$ in (8.17) one can make

$$
W=0 .
$$

As before, let us discuss these cases separately.

a) $\mathscr{H}_{\mathrm{C}}(A)=\mathscr{A}(+, 3)$

Referring to the results of the previous section, our first observation is that all $(A, \sigma)$ defined by (10.4) satisfy the local conditions for the existence of the real degeneracy. In fact, Eqs. (9.27), (9.28), and (9.31) with

$$
k^{2}=1+\frac{b(u)}{\alpha^{2}}
$$

and for an arbitrary real function $b(u)$ are satisfied not only in an open set but on the whole of $\Sigma$. Moreover, it turns out that the whole real degeneracy in $[(A, \sigma)]$ is automatically contained in the constraint surface. Namely, let us express $\left[\left(A^{\prime}, \sigma^{\prime}\right)\right] \in$ $\operatorname{Deg}_{\mathbb{R}}[A, \sigma]$ given by (9.24) in terms of (10.4). We obtain

$$
H^{\prime}=H+b(u), \quad W^{\prime}=W, \quad \phi^{\prime}=\phi .
$$

In fact, one can easily check that $H^{\prime}, W^{\prime}$ satisfy (8.17-8.20) hence the metric tensor ${ }^{4} \mathrm{~g}$ is again a vacuum solution to Einstein's equations. What is not completely clear 
is whether a function $k$ of the form (10.7) can be defined globally - by a suitable choice of $b-$ on $\Sigma$ even when $z, u$ and consequently $W, Z, H, \alpha$ are defined only locally. We can claim for certain that in a case when $z, u$ are defined globally, then $\operatorname{Deg}_{\mathbb{R c o n}}[A, \sigma]$ consists of $\left[\left(A^{\prime}, \sigma^{\prime}\right)\right]$ as in Statement 9.1(a) with $k$ of the form (10.7).

An important question is whether the degeneracy $\operatorname{Deg}_{\mathbb{R} c o n}[A, \sigma]$ is transversal to orbits of the group generated by diffeomorphisms and scalar constraints. Can $\left[\left(A^{\prime}, \sigma^{\prime}\right)\right] \in \operatorname{Deg}_{\mathbb{R} \text { con }}[A, \sigma]$ be reached from $(A, \sigma)$ by using diffeomorphism, and time evolution? The simplest way to find an answer is to go to the 4-dim space-time. Then $\left(A^{\prime}, \sigma^{\prime}\right)$ and $(A, \sigma)$ correspond to

$$
g^{\prime}=2 d z d \bar{z}-2 d u[d r+W d z+\bar{W} d \bar{z}+(H+b(u)) d u]
$$

and to

$$
g=2 d z d \bar{z}-2 d u(d r+W d z+\bar{W} d \bar{z}+H d u)
$$

respectively, where $b(u)$ is an arbitrary real function and $W, H$ are as in (8.17-8.20). Suppose

$$
g^{\prime}=\phi^{*} g
$$

$\phi$ being a space-time diffeomorphism. Then $\phi_{*} \frac{\partial}{\partial r}$ and $\frac{\partial}{\partial r}$ are both triple directions of the Weyl tensor. Thus

$$
\begin{aligned}
\phi_{*} \frac{\partial}{\partial r} & \sim \frac{\partial}{\partial r} . \\
\phi^{*} d u & \sim d u .
\end{aligned}
$$

[The equation for $\phi^{*} d u$ follows also from the particular form of $\phi^{*} g$ (10.10).] Consider now the infinitesimal diffeomorphism

$$
\mathscr{L}_{X} g=b(u)(d u)^{2} .
$$

From terms $d z d z, d z d \bar{z}, d u d r$ we find restrictions for a vector field $X$,

$$
\begin{aligned}
X= & A(u) \frac{\partial}{\partial u}+\left[i B_{1}(u) z+B_{0}(u)\right] \frac{\partial}{\partial z}+\left[-i B_{1}(u) \bar{z}+\bar{B}_{0}(u)\right] \frac{\partial}{\partial \bar{z}} \\
& +\left[-A_{u}(u) r+E(z, \bar{z}, u)\right] \frac{\partial}{\partial r}
\end{aligned}
$$

where $B_{1}$ is a real function of $u$. From other terms we get the set of equations, involving $X, W, H$ which can be satisfied only for very special cases of $W, H$, and $b(u)$. Therefore, almost all the transformations (10.8) are transversal to the fibers defined on the constrained surface by the constraints.

b) $\mathscr{H}_{\mathbf{C}}=\mathscr{b}(+)$

These solutions are among the special cases for which there exists a 4-dimensional diffeomorphism which carries $(10.9)$ into $\left(10.9^{\prime}\right)$. But now there appear new transformations $(A, \sigma) \rightarrow\left(A^{\prime}, \sigma^{\prime}\right)$ leaving $\left(T^{0}, T^{1}\right)$. 
Expressions (10.4) for $A$ and $\sigma$ can be written now in the following form:

$$
\begin{gathered}
A^{+}=\frac{i}{2 \alpha}\left(h_{\bar{z}} d u+d \phi_{\bar{z}}\right), \quad A^{3}=\frac{i}{2} d \ln \alpha \\
\sigma=\frac{1}{2}\left[\tau^{+} \alpha \frac{\partial}{\partial \bar{z}}+\tau^{-} \alpha \frac{\partial}{\partial z}-\tau^{3}\left(\frac{\partial}{\partial u}+\phi_{\bar{z}} \frac{\partial}{\partial z}+\phi_{z} \frac{\partial}{\partial \bar{z}}\right)\right] \\
\alpha^{2}=-\phi_{z} \phi_{\bar{z}}-h-\bar{h}-\phi_{u}
\end{gathered}
$$

with respect to the coordinates $(\operatorname{Re} z, \operatorname{Im} z, u), h$ and $\phi$ being a holomorphic and a real function respectively. The set $\operatorname{Deg}_{\mathbb{R} c o n}[A, \sigma]$ consists of those $\left[\left(A^{\prime}, \sigma^{\prime}\right)\right] \in \operatorname{Deg}_{1}[A, \sigma]$ given by Statement 9.1(b) which satisfy both the reality conditions and the constraints. One can find that a general form of such $A^{\prime}$ and $\sigma^{\prime}$ is given by substitution of

$$
\begin{gathered}
z^{\prime}=\frac{c z}{\dot{V}}, \quad u^{\prime}=V(u), \quad \dot{V}:=\frac{d}{d u} V, \\
h^{\prime}=\frac{|c|^{2} h}{\dot{V}^{2}}+f(u) z+\bar{f}(u) \bar{z}+e(u)
\end{gathered}
$$

and $\phi^{\prime}$ being arbitrary real function, into (10.14) where $c \in \mathbb{C}$ is a constant, $f$ is a complex function of $u$ and $e, V$ are real functions of $u$. Arbitrariness of $\phi^{\prime}$ means that in this case observables $\left(T^{0}, T^{1}\right)(A, \sigma)$ don't depend on Hamiltonian evolution given by the change of the function $\phi$ in (10.1) which defines a surface $\Sigma$ in the space-time (8.14). Transformations of $h^{\prime}$ are transversal to orbits of the transformation group generated by constraints. For example

$$
\begin{gathered}
\sigma^{\prime}=k\left(\sigma^{+} \tau_{+}+\sigma^{-} \tau_{-}\right)+k^{2} \sigma^{3} \tau_{3}, \\
A^{\prime}=\frac{1}{k} A^{+} \tau_{+}+A^{3} \tau_{3}, \\
k=\mathrm{const}
\end{gathered}
$$

correspond up to a certain diffeomorphism to the rescaling

$$
g^{\prime}=k^{2} g
$$

and leads to a non-equivalent $g^{\prime}$ except when $g$ admits a homothetical Killing vector field.

\section{Concluding Remarks}

In our work "degeneracy" means that there are inequivalent initial data which correspond to the same value of the loop variables. The main result of our paper is the finding of all the points in the space of initial data for classical gravity (as well as in the whole phase space for complex gravity) at which the loop variables become degenerate and characterizing their degeneracy. We found that those points correspond to vacuum space-times admitting a covariantly constant null direction. There are two classes of such points: (i) when the holonomy group is the 4-dimensional group of the null rotations, (ii) when the holonomy group consists of some null rotations but is 2-dimensional. In the second case space-time admits a covariantly constant vector field and is known as a p.p. wave [15]. The surfaces of degeneracy of the loop functionals are different for each of those cases. 
It turns out that the degeneracy cannot be removed by the factoring of the phase space by the constraint transformations group. It seems to suggest that the loop variables may not give a sufficiently good parametrization of the phase space for gravity. However, the problem arises with regard to the physical meaning of the resulting gravitational fields. Although it is not proven in our work or in the literature it seems that such space-times cannot be asymptotically flat and that they do not admit a compact surface of initial data. This conjecture should be examined better. In any case, the degenerate solutions are probably of "measure" zero in the space of solutions. Their importance rests in the possibility that they may separate the solutions space into regions with different physical properties.

As our results concerning the $T$-variables on the extended phase space $\Gamma$ for complexified gravity (on a real 4-manifold) show the essential feature of $(A, \sigma)$ is the holonomy algebra of $A$, more specifically, the complexification $\mathscr{H}_{\mathrm{C}}$ of the holonomy algebra. Another and independent result of our work is the theorem which states that $\mathscr{H}_{\mathrm{C}}$ is preserved under Hamiltonian evolution if the triad is linearly independent. The $\mathscr{H}_{\mathrm{C}}$ may be interpreted now as the first known observable in general relativity theory. On the other hand, we have given an example that when the triad is linearly dependent, time evolution changes $\mathscr{H}_{\mathrm{C}}$. This shows that a generalization of our results to the (also interesting) case of linearly dependent triads is not direct. It is remarkable, however, that even in the example presented, the degeneracy class of $(A, \sigma)$ (according to the classification in Theorem 6.1) is the same.

Another important issue is the examination of the derivatives of the loop variables. One may ask for which points $(A, \sigma)$ there exist directions $(\delta A, \delta \sigma)$ tangent to $\Gamma$ such that the variation of $\left(T^{0}, T^{1}\right)$ is identically zero for all the loops in the 3-manifold? What may occur at such a point can be illustrated by the following example. Suppose $A=A^{+} \tau_{+}$and $\delta A=\delta A^{3} \tau_{3}$. One can check that the variation of $T^{0}$ is zero for any given loop. One may be surprised, however, for $T^{0}$ distinguishes between connections of the holonomy algebra spanned by $\left(\tau^{3}, \tau_{+}\right)$and those of the holonomy algebra given by multiples of $\tau_{+}$. But the difference turns out to be of the second order in $\delta A^{3}$. This question will be treated in a following paper [16].

There are situations (e.g., in $2+1$ gravity and in coupled harmonic oscillators [3]) in which degeneracy imply the existence of super selected sectors in the Hilbert space. That situation may also occur with respect to the degeneracies discussed in this paper.

Acknowledgements. The authors wish to thank Joseph Samuel for initial discussions about this problem. During the course of this research various technical points were also discussed with Lee Smolin and Charles Torre. We are particularly grateful to Abhay Ashtekar for several important discussions and for emphasizing the generality of our investigation.

J. Lewandowski was supported by the Fulbright Program through the Counsel for International Exchange of Scholars and in part by the Polish Comitee for Scientific Research (KBN) through grant no. 204309101. The research of J. N. Goldberg was supported by the NSF through Grant No. PHY-9005740.

\section{References}

1. Ashtekar, A.: Phys. Rev. Lett. 57, 2244-2247 (1986)

2. Ashtekar, A.: New perspectives in canonical gravity. (Naples: Bibliopolis 1988)

3. Ashtekar, A.: Lectures on nonperturbative canonical gravity. (Notes prepared in collaboration with R. Tate), Singapore: World Scientific 1981 
4. Ashtekar, A.: Phys. Rev. D36, 1587-1603 (1987)

5. Jacobson, T., Smolin, L.: Nucl. Phys. B 229, 295 (1988)

6. Rovelli, C., Smolin, L.: Nucl. Phys. B 331, 80-152 (1990)

7. Ashtekar, A., Husain, V., Rovelli, C., Samuel, J., Smolin, L.: Class. Quantum Grav. 6, L 185 (1989)

8. Ashtekar, A., Rovelli, C.: Loop representation for the Maxwell field: quantum Faraday lines. Submitted to J. Math. Phys.

9. Ashtekar, A., Rovelli, C., Smolin, L.: Gravitons and loops. Preprint (1991)

10. Gambini, R., Brugmann, B., Pullin, J.: Knot invariants as nondegenerate states of four dimensional quantum gravity. Syracuse University Preprint, SU-GP-91/8-4 (1991)

11. Samuel, J.: Private communication (1991)

12. Schell, J.F.: J. Math. Phys. 2, 202 (1962)

13. Goldberg, J.N., Kerr, R.P.: J. Math. Phys. 2, 327 (1961)

14. Kerr, R.P., Goldberg, J.N.: J. Math. Phys. 2, 332 (1961)

15. Kramer, D. et al.: Exact solutions of Einstein's field equations. Deutscher Verlag der Wissenschaften 1980

16. Goldberg, J.N., Lewandowski, J.: In preparation

Communicated by S.-T. Yau 